\title{
El vivir solo se asoció a un aumento de la mortalidad en adultos jóvenes
}

Living alone was associated with increased mortality in young adults

Jacob A. Udell y col. Arch Intern Med 2012;172:1086-1094.

\section{Objetivos}

Determinar si la condición de vivir solo se asocia con un aumento del riesgo cardiovascular y de la mortalidad en pacientes con riesgo de, o con, aterotrombosis.

\section{Diseño y lugar}

Estudio multicéntrico de cohorte prospectiva realizado en 44 ciudades de América del Norte, América Latina, Europa Occidental, Europa del Este, Oriente Medio, Japón y otros países asiáticos.

\section{Pacientes}

Pacientes de 45 años o más con enfermedad coronaria, cerebrovascular o arteriopatía periférica establecida; y aquellos sin aterotrombosis, pero con al menos tres factores de riesgo para enfermedad cardiovascular (diabetes mellitus, microalbuminuria, índice tobillo brazo $<0,9$, estenosis carotidea asintomática de al menos el $70 \%$, espesor medio intimal engrosado, hipertensión arterial, dislipemia, tabaquismo actual, edad mayor a 65 años en hombres y 70 en mujeres).

\section{Medición de resultados principales}

El resultado primario fue muerte por todas las causas. Como resultados secundarios, se consideraron muerte de causa cardiovascular y un indicador combinado de muerte de causa cardiovascular, infarto agudo de miocardio (IAM) no fatal y accidente cerebrovascular (ACV) no fatal.

\section{Resultados principales}

Se observó un incremento de la mortalidad en las personas que viven solas comparándolas con las que viven acompañadas, siendo los resultados más significativos en el subgrupo etareo de entre 45 y 65 años de edad. En contraposición, no se observó aumento de la mortalidad en los mayores de 80 años que viven solos comparados con los que están acompañados. Una tendencia similar se presentó en la asociación entre la variable edad y el vivir solo, con el riesgo de muerte cardiovascular (ver tabla 1).

Tabla 1: Incidencia de eventos de mortalidad por cualquier causa, muerte cardiovascular, por infarto agudo de miocardio y por enfermedad cerebrovascular, según la condición de vivir solos.

\begin{tabular}{|c|c|c|c|c|}
\hline $\begin{array}{l}\text { Resultados a } 4 \text { años de } \\
\text { seguimiento }\end{array}$ & $\begin{array}{c}\text { Eventos en personas que viven } \\
\text { solos (\%) } \\
\text { (n: 8.594) }\end{array}$ & $\begin{array}{c}\text { Eventos en personas que viven } \\
\text { acompañadas (\%) } \\
\text { (n: } 35.979)\end{array}$ & Edad & $\begin{array}{l}\text { Hazard ratio ajustado* } \\
\text { (IC 95\%) }\end{array}$ \\
\hline Mortalidad & 14,1 & 11,1 & $\begin{array}{c}45 \text { a } 65 \\
66 \text { a } 80 \\
\text { Mayor a } 80\end{array}$ & $\begin{array}{l}1,24(1,01 \text { a } 1,51) \\
1,12(1,01 \text { a } 1,26) \\
0,92(0,79 \text { a } 1,06)\end{array}$ \\
\hline Muerte cardiovascular & 8,6 & 6,8 & $\begin{array}{c}45 \text { a } 65 \\
66 \text { a } 80 \\
\text { Mayor a } 80\end{array}$ & $\begin{array}{l}1,29(1,01 \text { a } 1,64) \\
1,11(0,96 \text { a } 1,28) \\
0,93(0,77 \text { a } 1,12)\end{array}$ \\
\hline Resultado combinado** & 15,8 & 14,2 & No aplicable & $1,03(0,96$ a 1,12$)$ \\
\hline IAM & 3,8 & 3,7 & No aplicable & $1,01(0,87$ a 1,18$)$ \\
\hline ACV & 5,5 & 5,4 & No aplicable & $0,98(0,86$ a 1,12$)$ \\
\hline
\end{tabular}

*Ajuste por los siguientes factores de riesgo: edad, sexo, empleo, educación, etnia, área geográfica, historia de tabaquismo, diabetes mellitus, índice de masa corporal, fibrilación auricular, insuficiencia cardiaca, enfermedad vascular, evento isquémico previo, uso de estatinas y/o de aspirina. ${ }^{* *}$ Muerte cardiovascular, por infarto agudo de miocardio (IAM) o por accidente cerebro vascular (ACV).

En este estudio la condición de vivir solo se asoció con un incremento de la mortalidad siendo los resultados más significativos en el subgrupo de edades más jóvenes, sin embargo esta obser- vación requiere ser confirmada en próximos estudios.

Fuente de financiamiento/Conflicto de intereses: Sanofi Aventis, Bristol-Myers Squibb, Fundación Waksman.

\section{Comentario}

Existen resultados contradictorios en estudios previos al evaluar la asociación entre el grado de interacción social y mortalidad o eventos CV luego de un IAM $^{1-4}$. En este estudio, la asociación entre vivir solo y mortalidad global y por causa cardiovascular es particularmente consistente en los individuos de entre 45 y 65 años, y no tanto en el grupo de mayores de 80 años, los que a priori, se verían más afectados por la propia enfermedad. Cabe mencionar como limitación de este estudio que la variable de interés, el vivir solo, fué dicotomizada al inicio del estudio, por lo cual no podemos puntualizar si el riesgo es dinámico conforme pasa el tiempo. Además no hay información sobre potenciales confundidores como el autoreporte de síntomas depresivos, calidad de vida, diagnósticos psicosociales, estado civil, entre otros.

\section{Conclusiones del comentador}

La condición de vivir solo se asoció en forma independiente con un incremento del riesgo de mortalidad por cualquier causa y muerte cardiovascular. Los individuos jóvenes que viven solos parecen tener un curso más desfavorable en el desarrollo de enfermedad cardiovascular frente a individuos de mayor edad, hecho que hace suponer la influencia negativa del aislamiento y la falta de soporte social en el comportamiento en salud de esta población, lo cual debiera ser confirmado en próximos estudios de investigación.

David Colica [ Servicio de Medicina Familiar y Comunitaria del Hospital Italiano de Buenos Aires. david.dolica@hiba.org.ar]

Colica D. El vivir solo se asoció a un aumento de la mortalidad en adultos jóvenes. Evid Act Pract Ambul.2014 17(4). Oct-Dic 129. Comentado de: Udell JA, Steg PG, Scirica B y col. Living alone and cardiovascular risk in outpatients at risk of or with atherothrombosis. Arch Intern Med 2012;172:1086-1094. PMID: 22711020.

\section{Referencias}

1. Rosanski A y col. Impact of psychological factors on the pathogenesis of cardiovascular disease an implications for therapy. Cirulation 1999; 99(16):2192-2217. 2. Case RB y col. Living alone after myocardial infarction. Impact in prognosis. JAMA 1992;267(4):515-519.

3. O' Shea JC y col. Comparison of outcomes of patiens with myocardial infarction when livin alone versus those not living alone. Am J Cardiol. 12002;90(12):1374-1377. 4. Bucholz E, Krumholz H. Loneliness and Living Alone. Arch Intern Med. 2012;172(14):1084-1085. 